\title{
Effect of antioxidants on post thaw microscopic, oxidative stress parameter and fertility of Boer goat spermatozoa in Tris egg yolk glycerol extender.
}

\begin{abstract}
This study was conducted to determine the effect of antioxidants on standard semen parameters, lipid peroxidation and fertility of Boer goat semen after cryopreservation. Ejaculates from four bucks were collected, evaluated and pooled at $37 .{ }^{\circ} \mathrm{C}$. The pooled semen was diluted with Tris citric acid fructose for washing. Semen samples, which were diluted with a Tris-based extender containing the antioxidant ascorbic acid $(8.5 . \mathrm{mg} / \mathrm{ml})$, butylated hydroxytoluene $(2 . \mathrm{mM})$, cysteine $(5 . \mathrm{mM})$ and hypotaurine $(10 . \mathrm{mM})$ and an extender without antioxidant supplementation were cooled to $4 .{ }^{\circ} \mathrm{C}$ and frozen in 0.25 straws with programmable freezer and finally stored in liquid nitrogen. Data (10 replicates) were analyzed by one-way analysis of variance. Mean $( \pm$ SEM) progressive motility was significantly higher in ascorbic acid than other supplement groups and control samples (P) 0.05). Best values were observed in ascorbic acid followed by BHT, cysteine, and hypotaurine. Antioxidant supplementation in extender showed significant $(\mathrm{P}<0.05)$ better values than the control group for sperm membrane integrity, acrosome integrity and viability. The ability of antioxidants to reduce the lipid peroxidation (LPO) after freeze thawing was measured by the formation of malondialdehyde (MDA) using the thiobarbituric acid method. Results showed that addition of antioxidants significantly reduced the rate of LPO in comparison to control $(\mathrm{P}<0.05)$. Ascorbic acid exhibited better values $(1.27 \pm 0.28)$, than butylated hydroxytoluene, cysteine and hypotaurine $1.32 \pm 0.42,2.27 \pm 0.16$ and $2.38 \pm 0.17$ respectively, which are significantly better than control (3.52 \pm 0.54$)$. Higher pregnancy rate was observed with ascorbic acid followed by butylated hydroxtolune, hypotaurine and cysteine. However, differences in the fertility rate were non-significant with hypotaurine, cysteine and control groups
\end{abstract}

Keyword: Ascorbic acid; Boer goat; Butylated hydroxytoluene; Cryopreservation; Cysteine; Hypotaurine; Lipid peroxidation. 